\title{
Online computation of similarity between handwritten characters
}

\author{
Oleg Golubitsky and Stephen M. Watt
}

Department of Computer Science, University of Western Ontario, London Ontario, Canada

\begin{abstract}
We are interested in the problem of curve identification, motivated by problems in handwriting recognition. Various geometric approaches have been proposed, with one of the most popular being "elastic matching." We examine the problem using distances defined by inner products on functional spaces. In particular we examine the Legendre and Legendre-Sobolev inner products. We show that both of these can be computed in online constant time. We compare both with elastic matching and conclude that the Legendre-Sobolev distance measure provides a competitive alternative to elastic matching, being almost as accurate and much faster.
\end{abstract}

Keywords: handwriting recognition, real-time computing, online algorithms, elastic matching, orthogonal polynomials, Legendre polynomials, Sobolev inner products

\section{INTRODUCTION}

Most applications involving online handwriting recognition require the recognition to be performed in real time, without causing any delay after the pen is lifted up. Yet most techniques for handwriting recognition, including the most popular elastic matching ${ }^{1-3}$, must wait until the entire curve is traced out, before any analysis can start. This approach inevitably causes delays after the character is written. The problem becomes even more noticeable when the number of character classes is large, as is the case with mathematical handwriting recognition.

Char and Watt ${ }^{4}$ have shown that polynomially parametrized curves of degree 10 approximate most handwritten mathematical characters well enough to be visually indistinguishable from the originals. In another paper ${ }^{5}$, we have demonstrated that coefficients of such polynomials can be computed online, as the character is being written. More precisely, in terms of the online computational complexity used in our paper ${ }^{5}$, this computation requires a constant number of operations per each point in the curve, which can be performed as soon as that point arrives, and a constant-time overhead at the end, after the pen is lifted.

Polynomial parametrizations are convenient, because they allow to instantly compute distances between curves. The kind of distance depends on the choice of the orthogonal polynomial basis, with respect to which we compute the coefficients. For example, the Chebyshev basis corresponds to the maximal distance between the corresponding points on the curves, namely

$$
\max _{t \in[-1,1]}\left(x_{1}(t)-x_{2}(t)\right)^{2}+\left(y_{1}(t)-y_{2}(t)\right)^{2}
$$

the Legendre basis to the $L_{2}$ distance

$$
\int_{-1}^{1}\left(x_{1}(t)-x_{2}(t)\right)^{2}+\left(y_{1}(t)-y_{2}(t)\right)^{2} d t
$$

and the Legendre-Sobolev basis considered in this paper to the distance in the Sobolev space $H^{1}$ :

$$
\int_{-1}^{1}\left(x_{1}(t)-x_{2}(t)\right)^{2}+\left(y_{1}(t)-y_{2}(t)\right)^{2} d t+\int_{-1}^{1}\left(x_{1}^{\prime}(t)-x_{2}^{\prime}(t)\right)^{2}+\left(y_{1}^{\prime}(t)-y_{2}^{\prime}(t)\right)^{2} d t
$$

Further author information: (Send correspondence to Stephen M. Watt) Oleg Golubitsky: E-mail: oleg@scl.csd.uwo.ca, Telephone: +1 (519) 661-4296

Stephen M. Watt: E-mail: watt@csd.uwo.ca, Telephone: +1 (519) 859-2345 
The above formulas make it clear that the distance depends on the parametrization of the curve. We chose three parametrizations for comparison: by time, by arc length, and by affine arc length. ${ }^{6}$

In this paper, we verify experimentally that for the purpose of recognition of handwritten mathematical characters, the distance between Legendre-Sobolev coefficient vectors is more robust than the distance between Legendre coefficients. We show examples of characters, which are wrongly classified as similar by the Legendre distance. Moreover, we demonstrate that the Legendre-Sobolev distance measure is almost as accurate as the elastic matching distance. This conclusion is valid for all three parametrizations. Also, for any fixed distance measure, parametrization by arc length shows a slight advantage over the other two parametrizations.

\section{LEGENDRE AND LEGENDRE-SOBOLEV INNER PRODUCTS}

The Legendre inner product between two functions $f, g:[-1,1] \rightarrow \mathbb{R}$ is defined by

$$
\langle f, g\rangle_{L}=\int_{-1}^{1} f(t) g(t) d t
$$

and the Legendre-Sobolev inner product ${ }^{7}$ is given by

$$
\langle f, g\rangle_{L S}=\int_{-1}^{1} f(t) g(t) d t+\lambda \int_{-1}^{1} f^{\prime}(t) g^{\prime}(t) d t
$$

where $\lambda$ is a positive real parameter. The latter may involve terms corresponding to higher order derivatives, but for the purposes of this paper we restrict ourselves to the first order. We also use $\lambda=1$.

Systems of orthogonal polynomials $\left\{P_{0}, P_{1}, P_{2}, \ldots\right\}$ with respect to the above inner products can be obtained by applying the Gram-Schmidt orthogonalization process to the monomial basis $\left\{1, t, t^{2}, \ldots\right\}$. A function $f:[-1,1] \rightarrow \mathbb{R}$ can be represented as an infinite linear combination of the orthogonal polynomials

$$
f(t)=\sum_{i=0}^{\infty} a_{i} P_{i}(t)
$$

called the Legendre (or Legendre-Sobolev) series, depending on the choice of the inner product. The coefficients of the series are given by the formulas

$$
a_{i}=\frac{\left\langle f, P_{i}\right\rangle}{\left\langle P_{i}, P_{i}\right\rangle}, \quad i=0,1,2, \ldots
$$

Here $\langle\cdot, \cdot\rangle$ stands for one of the above inner products $\langle\cdot, \cdot\rangle_{L}$ or $\langle\cdot, \cdot\rangle_{L S}$ and it is assumed that the integrals involved in the inner product are well defined for $f$.

Truncation of the above series at degree $d$

$$
\sum_{i=0}^{d} a_{i} P_{i}(t)
$$

gives a degree $d$ polynomial which is the closest to the function $f$ among all polynomials of degree $d$, in terms of the Euclidean norm $\|f\|=\sqrt{\langle f, f\rangle}$ induced by the inner product. Thus, the truncated series is an approximation of the function $f$ :

$$
f(t) \approx \sum_{i=0}^{d} a_{i} P_{i}(t)
$$

Such approximation allows one to think of functions as points in a $(d+1)$-dimensional vector space and compute the distance between two functions $f$ and $g$ as a Euclidean distance between their coefficient vectors. Namely, if

$$
f(t) \approx \sum_{i=0}^{d} a_{i} P_{i}(t) \text { and } g(t) \approx \sum_{i=0}^{d} b_{i} P_{i}(t)
$$


then the distance between $f$ and $g$ is given by

$$
\|f-g\| \approx \sqrt{\sum_{i=0}^{d}\left(a_{i}-b_{i}\right)^{2} .}
$$

\section{COMPUTING DISTANCES IN REAL TIME}

An online algorithm for computing the coefficients of the truncated Legendre series has been proposed previously ${ }^{5}$. Here we summarize the idea and note that it is equally applicable to Legendre-Sobolev series.

Having precomputed the coefficients of orthogonal polynomials and squares of their norms $\left\langle P_{i}, P_{i}\right\rangle$, we use formula (1) to obtain the coefficients of the truncated series. Our function $f(t)$ is one of the two coordinate functions $x(t)$ and $y(t)$, whose values are sampled by the pen and arrive at time moments $t_{0}, t_{1}, t_{2}, \ldots$ with relatively constant time intervals. When the $k$-th point arrives, we update the values of the moments

$$
m_{i}=\int_{t_{0}}^{t_{k}} t^{i} f(t) d t, \quad i=0,1, \ldots d .
$$

This requires $O(d)$ operations for each point.

When the last point arrives at time $t_{N}$ and pen is lifted up, we apply linear substitution $\left[t_{0}, t_{N}\right] \rightarrow[-1,1]$ to rescale the moments to the interval $[-1,1]$ and change the basis from the monomial basis $\left\{1, t, t^{2}, \ldots t^{d}\right\}$ to the orthogonal polynomial basis $\left\{1, P_{1}, P_{2}, \ldots, P_{d}\right\}$. The change of basis requires $O\left(d^{2}\right)$ operations, independent of $N$. The value of $d$ is small, typically 10 .

In contrast, computation of the elastic matching distance requires $O(N)$ operations per point. Moreover, we usually need to normalize the size of the character before doing elastic matching, which implies that we cannot begin elastic matching before the pen is lifted and imposes $O\left(N^{2}\right)$ computational overhead after that.

For Legendre-Sobolev inner product, the same algorithm can be applied as for the Legendre inner product. In order to avoid computing the derivative of $f(t)$ at the points $t_{i}$, we use integration by parts:

$$
\begin{gathered}
\int_{t_{0}}^{t_{k}} t^{i} f(t) d t+\lambda \int_{t_{0}}^{t_{k}} i t^{i-1} f^{\prime}(t) d t=\int_{t_{0}}^{t_{k}} t^{i} f(t) d t+\lambda\left(\left.i t^{i-1} f(t)\right|_{t_{0}} ^{t_{k}}-\int_{t_{0}}^{t_{k}} i(i-1) t^{i-2} f(t) d t\right)= \\
\int_{t_{0}}^{t_{k}}\left[t^{i}-\lambda i(i-1) t^{i-2}\right] f(t) d t+\left.\lambda i t^{i-1} f(t)\right|_{t_{0}} ^{t_{k}}
\end{gathered}
$$

\section{COMPARISON WITH ELASTIC MATCHING}

Our experiments were performed on a data set of handwritten mathematical symbols collected at the Ontario Research Centre for Computer Algebra (ORCCA). The collection was taken from 16 test users, who were asked to provide handwritten samples of different mathematical symbols. The users were asked to provide samples only for characters that are familiar to them. Consequently, some characters have more samples than others. Out of all samples, we selected 1666 single-stroke characters. These characters were visually inspected and 1587 of them were classified into 107 different classes. The remaining 79 characters were not attributed to any class. A summary of the resulting class sizes is given in Figure 1. 

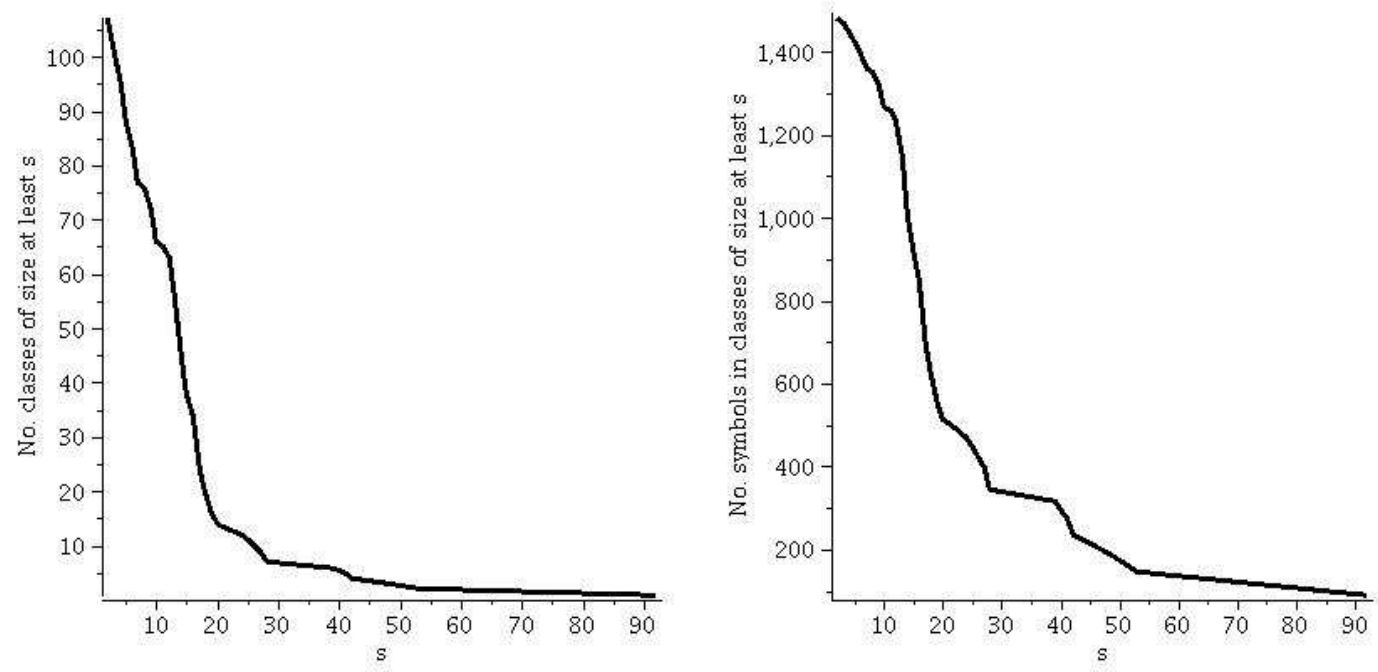

Figure 1. Numbers of classes of size at least $s$ (left) and total numbers of elements in these classes (right).

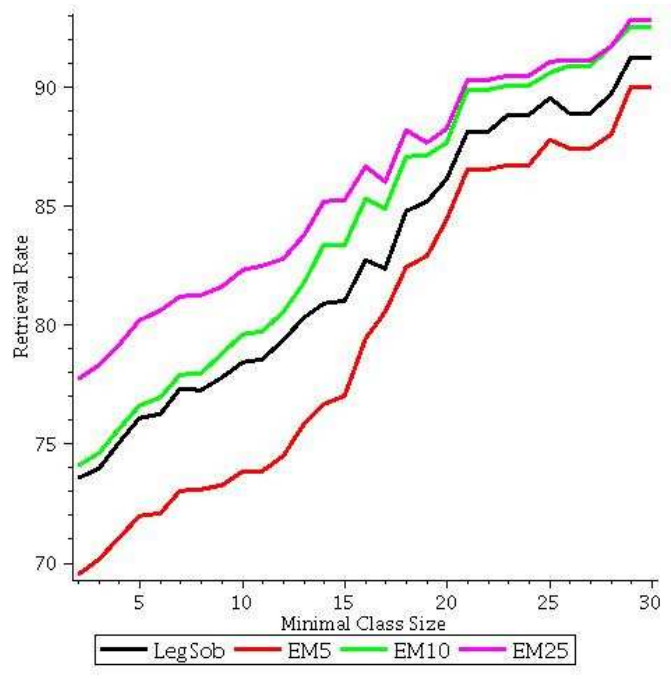

\begin{tabular}{l|cccccccc} 
& 2 & 5 & 10 & 15 & 20 & 25 & 30 & 40 \\
\hline LegSob & 73.5 & 76.1 & 78.4 & 81.0 & 86.1 & 89.5 & 91.3 & 91.5 \\
EM5 & 69.5 & 72.0 & 73.8 & 77.0 & 84.4 & 87.8 & 90.0 & 89.7 \\
EM10 & 74.1 & 76.6 & 79.6 & 83.4 & 87.7 & 90.6 & 92.5 & 92.9 \\
EM25 & 77.8 & 80.2 & 82.3 & 85.2 & 88.2 & 91.0 & 92.8 & 92.9
\end{tabular}

Table 1. Correct retrieval rates by nearest neighbor for Legendre-Sobolev distance and elastic matching distance with 5,10 , and 25 points, calculated for characters drawn from classes of size at least $s$, where $s$ ranges from 2 to 40 . Parameterization of curves by arc length is used for all distance measures. 


\subsection{Legendre-Sobolev versus Elastic Matching}

Our main experiment shows that classification accuracies of the Legendre-Sobolev distance measure and elastic matching are similar, while the Legendre-Sobolev distance can be computed much faster.

For each type of distance measure and every integer $s=2, \ldots, 40$, we computed the percentage of characters from classes of size at least $s$ that are correctly classified by their nearest neighbor (the latter is chosen among all 1666 characters in the database). Note that the elastic matching distance measure depends on an arbitrary parameter, the number of points that are sampled from the curve and used as input to the dynamic programming elastic matching algorithm. In our experiments we let this number of points vary. The results are summarized in Table 1.

For classes with few samples, elastic matching performs about $4 \%$ better than Legendre-Sobolev. For example, for classes with 15 samples we have $85.2 \%$ correct retrieval rate for elastic matching and $81.0 \%$ for LegendreSobolev. Naturally, the correct retrieval rate grows with the number of samples, regardless of the choice of the distance measure. The growth is nearly linear until the class size reaches about 25 . At this point, the correct retrieval rate levels off at about $90-93 \%$ for both distance measures, the elastic matching having only a $1-1.5 \%$ advantage over Legendre-Sobolev.

This advantage is not very significant, taking into account the fact that Legendre-Sobolev distance can be computed much faster. Indeed, computing Legendre-Sobolev distance for degree 10 amounts to adding 20 square differences (the Legendre-Sobolev expansion of degree 10 actually has 11 coefficients, but the constant term is eliminated as a result of normalization with respect to translation). Computation of elastic matching with 10 points amounts to 200 square differences (i.e., is 10 times slower than Legendre-Sobolev); and for 25 points, which we show below to be an optimal number of points for elastic matching, we have $25^{2} \times 2=1250$ square differences, which is more than 60 times slower than Legendre-Sobolev.

In practice, faster distance algorithm allows us to compare against a larger set of samples. As our experiments confirm, this directly translates into an increased classification accuracy.

The remaining experiments investigate in detail alternative choices of parameters that were fixed in the main experiment, such as parameterization of the curve, number of points for elastic matching, and number of nearest neighbors.

\subsection{Number of Points for Elastic Matching}

We show experimentally that the optimal number of points to be sampled from the character curve for elastic matching is about 25 (for the purpose of comparison of handwritten mathematical symbols). Table 2 shows the correct retrieval rates by nearest neighbor with respect to the elastic matching distance versus minimal allowed class size, for the number of sample points equal to $5,10,25,50$, and 100 .

\subsection{Choice of Parameterization}

We show experimentally that the optimal choice of curve parametrization is arc length, regardless of the distance measure. Table 3 an Table 4 show how the correct retrieval rates depend on the choice of parameterization, for the Legendre-Sobolev and elastic matching distance measures, respectively. We have examined three parameterizations that frequently appear in the literature: the natural parameterization by time, the parameterization by arc length, and the parameterization by affine arc length. The last two parameterizations depend only on the curve, whereas the first one also depends on the variations in speed with which the curve is being traced out. It is probably this latter dependence, that makes the distances based on the time parameterization less robust. For the Legendre-Sobolev distance measure, parameterizations by arc length and affine arc length yield similar recognition rates; for elastic matching, arc length parameterization is noticeably better.

\subsection{Number of Nearest Neighbors}

For classes with few elements, classification by the single nearest neighbor gives best results. As the size of classes increases, higher-order nearest neighbor methods gradually catch up (for Legendre-Sobolev) or take over (for elastic matching). The results are shown in Table 5 (for Legendre-Sobolev distance measure) and Table 6 (for elastic matching with 25 points). In all cases, curves are parameterized by arc length. 


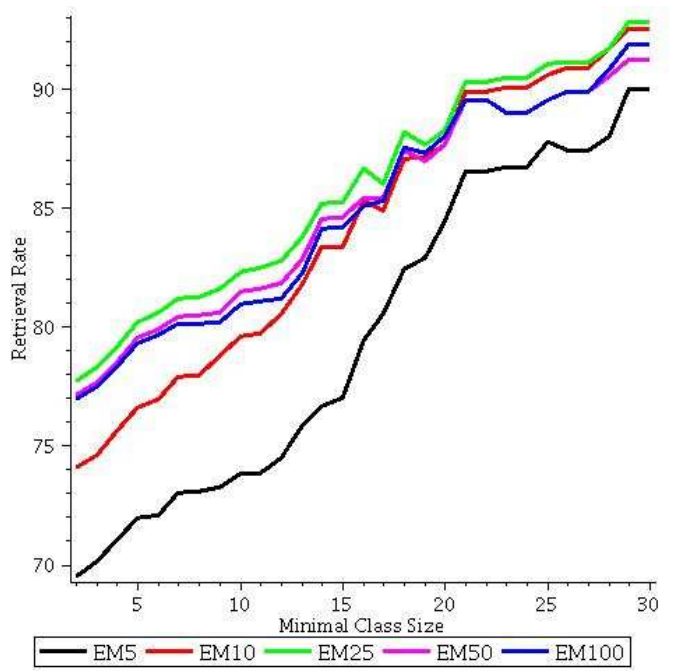

\begin{tabular}{l|cccccccc} 
& 2 & 5 & 10 & 15 & 20 & 25 & 30 & 40 \\
\hline EM5 & 69.5 & 72.0 & 73.8 & 77.0 & 84.4 & 87.8 & 90.0 & 89.7 \\
EM10 & 74.1 & 76.6 & 79.6 & 83.4 & 87.7 & 90.6 & 92.5 & 92.9 \\
EM25 & 77.8 & 80.2 & 82.3 & 85.2 & 88.2 & 91.0 & 92.8 & 92.9 \\
EM50 & 77.1 & 79.5 & 81.5 & 84.6 & 87.7 & 89.5 & 91.3 & 91.1 \\
EM100 & 76.9 & 79.3 & 81.0 & 84.2 & 88.0 & 89.5 & 91.9 & 91.5
\end{tabular}

Table 2. Retrieval rates by nearest neighbor for elastic matching distance with 5, 10, 25, 50, and 100 points, calculated for characters drawn from classes of size at least $s$, where $s$ ranges from 2 to 40. Parameterization of curves by arc length is used.

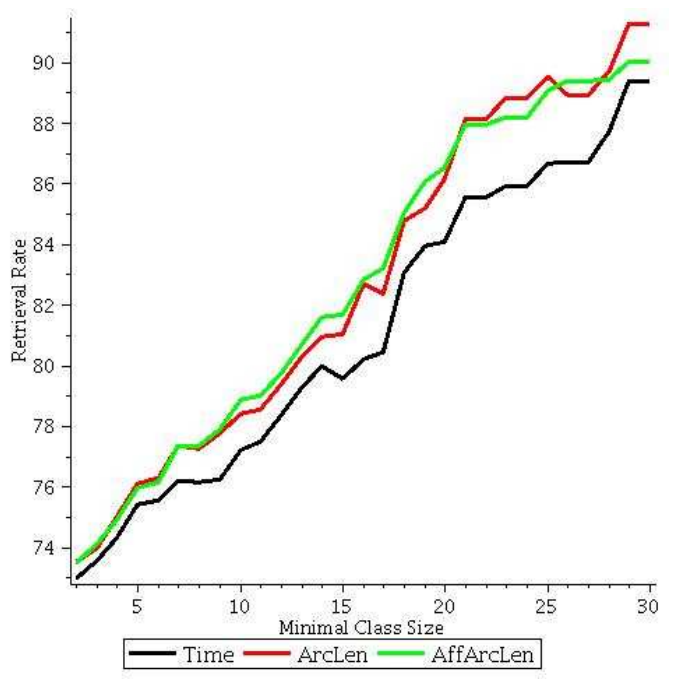

\begin{tabular}{l|cccccccc} 
& 2 & 5 & 10 & 15 & 20 & 25 & 30 & 40 \\
\hline Time & 73.0 & 75.4 & 77.2 & 79.6 & 84.1 & 86.7 & 89.4 & 91.1 \\
ArcLen & 73.5 & 76.1 & 78.4 & 81.0 & 86.1 & 89.5 & 91.3 & 91.5 \\
AffArcLen & 73.5 & 76.0 & 78.9 & 81.7 & 86.5 & 89.1 & 90.0 & 90.0
\end{tabular}

Table 3. Retrieval rates by nearest neighbor for Legendre-Sobolev distance and parameterizations by time, arc length, and affine arc length. 


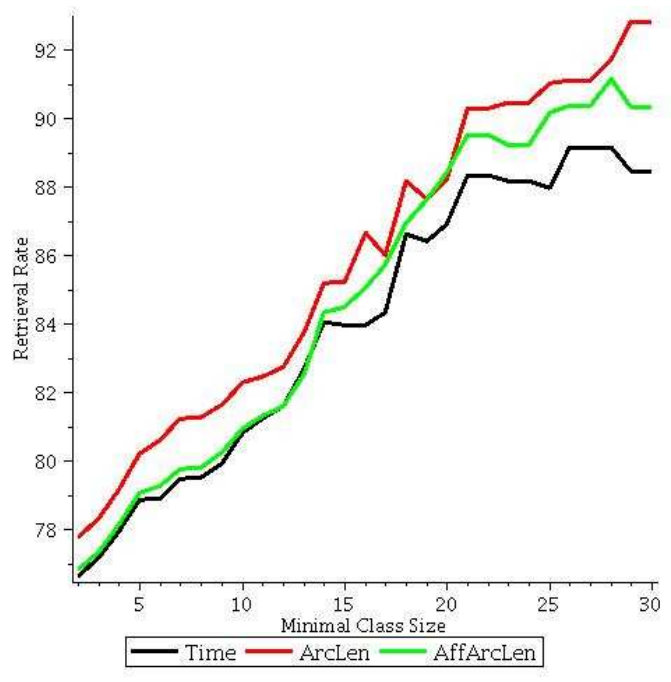

\begin{tabular}{l|cccccccc} 
& 2 & 5 & 10 & 15 & 20 & 25 & 30 & 40 \\
\hline Time & 76.6 & 78.9 & 80.8 & 84.0 & 86.9 & 88.0 & 88.5 & 89.3 \\
ArcLen & 77.8 & 80.2 & 82.3 & 85.2 & 88.2 & 91.0 & 92.8 & 92.9 \\
AffArcLen & 76.8 & 79.1 & 81.0 & 84.5 & 88.4 & 90.2 & 90.3 & 90.0
\end{tabular}

Table 4. Retrieval rates by nearest neighbor for elastic matching distance with 25 points and parameterizations by time, arc length, and affine arc length.

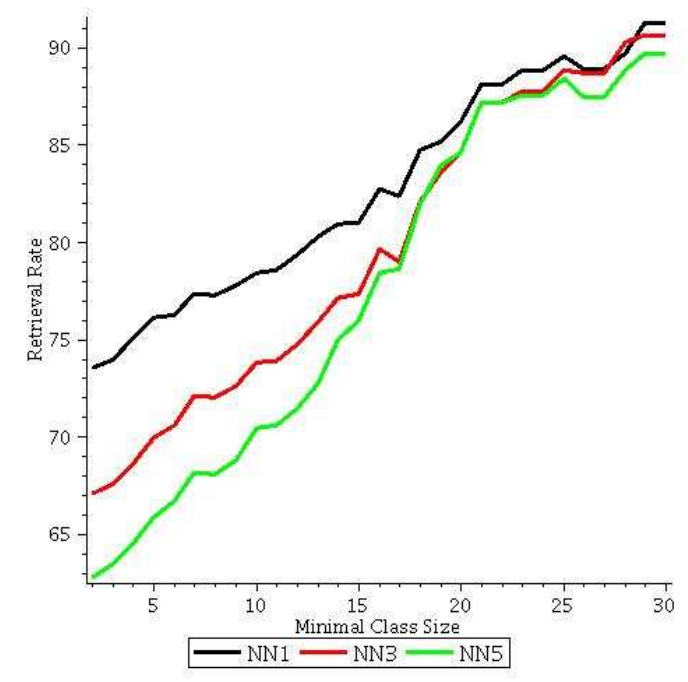

\begin{tabular}{c|cccccccc} 
& 2 & 5 & 10 & 15 & 20 & 25 & 30 & 40 \\
\hline NN1 & 73.5 & 76.1 & 78.4 & 81.0 & 86.1 & 89.5 & 91.3 & 91.5 \\
NN3 & 67.0 & 69.9 & 73.8 & 77.3 & 84.6 & 88.9 & 90.7 & 90.7 \\
NN5 & 62.8 & 65.8 & 70.5 & 76.0 & 84.6 & 88.4 & 89.7 & 90.4
\end{tabular}

Table 5. Retrieval rates by 1,3 , and 5 nearest neighbors for Legendre-Sobolev distance. Parameterization by arc length. 


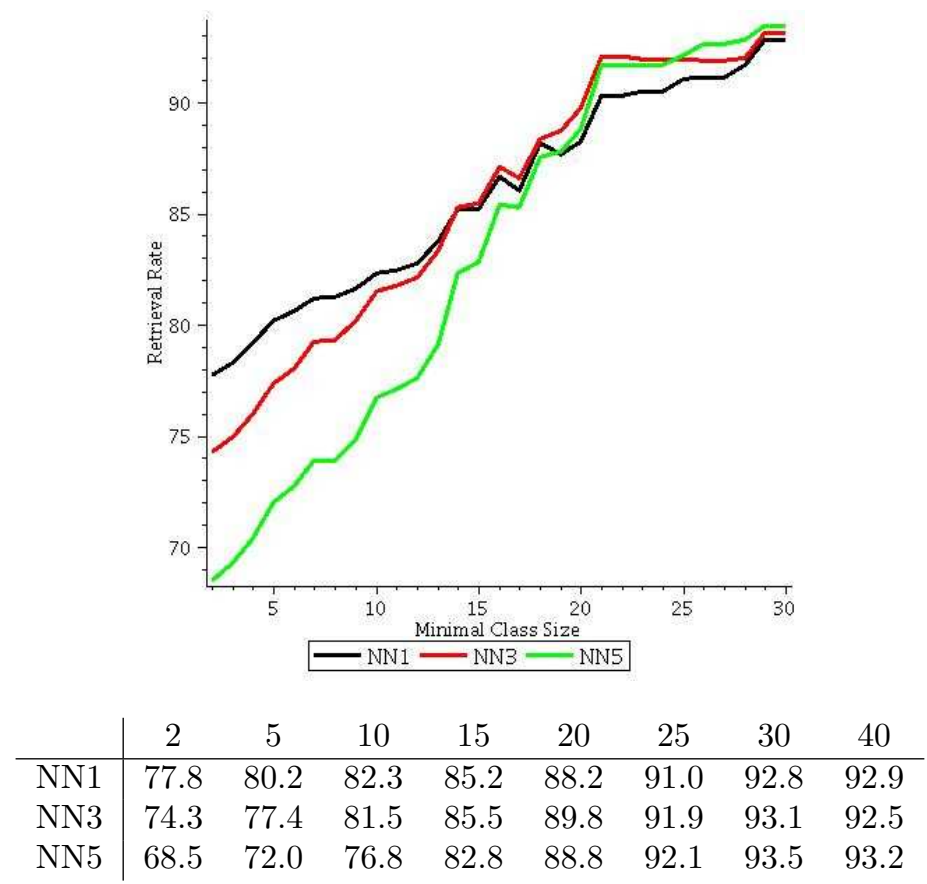

Table 6. Retrieval rates by 1,3 , and 5 nearest neighbors for Legendre-Sobolev distance. Parameterization by arc length.

\subsection{Legendre Distance Shows Poor Performance}

As Table 7 shows, the performance of the Legendre distance measure is inferior to that of Legendre-Sobolev distance, and even to that of elastic matching with only 5 sample points. As Legendre and Legendre-Sobolev distances require the same numbers of arithmetic operations, we conclude that the latter is preferable to the former for the purposes of recognition of handwritten mathematical symbols.

Examples of the 25 closest symbols to a character " $m$ " with respect to the three distance measures and parametrization by arc length are shown in Figures 2 and 3.

\section{ON THE PERFORMANCE OF DISTANCE MEASURES REQUIRED FOR RECOGNITION OF HANDWRITTEN MATHEMATICAL SYMBOLS}

Recognizers for certain settings, e.g. mathematical handwriting, may have to classify a character among about 200 classes. Our experiments have shown that at about 25-30 sample characters per class the performance of various distance measures stabilizes; to be safe, assume that we need 50 sample characters per class. Then, with the Legendre-Sobolev distance measure, whose computation amounts to adding 20 square differences (i.e., performing 60 arithmetic operations), we can compare a given character with all model characters in our database in $200 \times 50 \times 60=300,000$ arithmetic operations.

The computation of Legendre-Sobolev coefficients from moments requires floating point arithmetic. ${ }^{5}$ However, this can be done in advance for each character in the database, and needs to be done only once for the character to be classified. Once the Legendre-Sobolev coefficients are obtained, the distance can be computed in 16-bit integer arithmetics.

Indeed, the absolute values of the Legendre-Sobolev coefficients for character curves in our database, after size normalization, do not exceed 1. Moreover, as we will show below, we only need to know the coefficients up to four decimal digits. Thus we can multiply each coefficient by 10000 , round the result to the nearest integer, and safely use it in distance computations instead of the original floating point value. At present, it should be possible to perform 300,000 16-bit arithmetic operations almost instantly on even inexpensive modern hardware. 


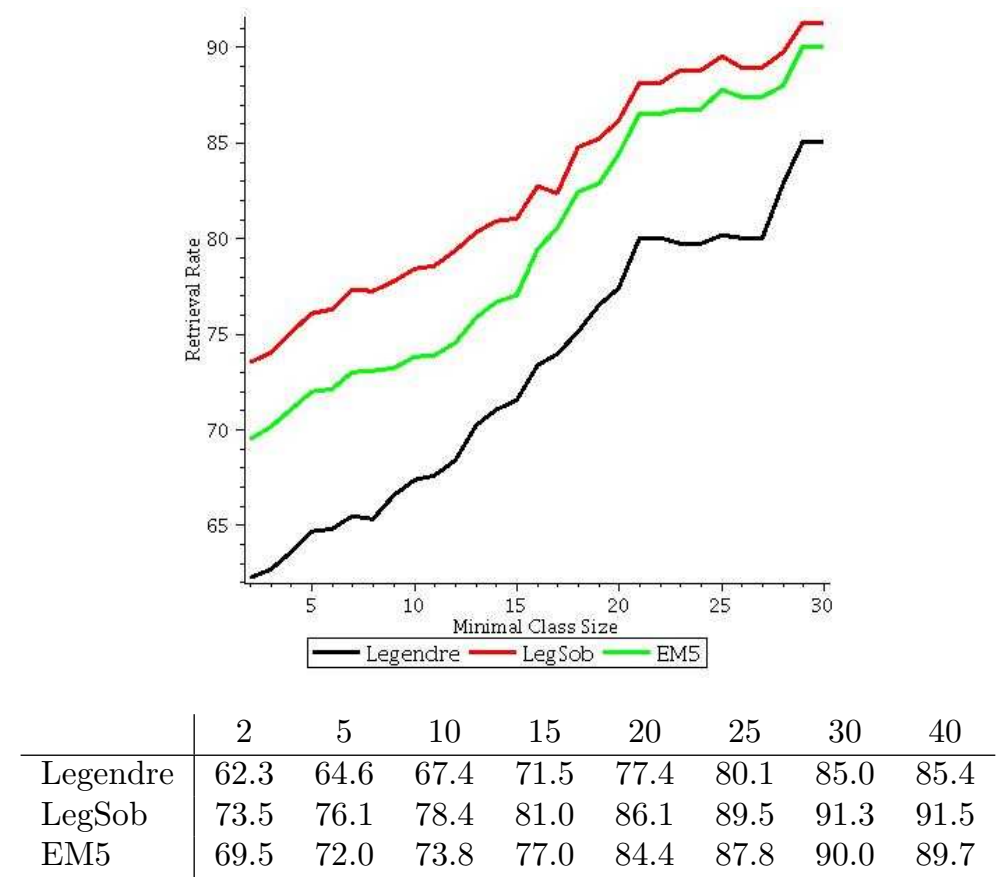

Table 7. Retrieval rates by nearest neighbor for Legendre, Legendre-Sobolev, and 5-point elastic matching distance measures. Parameterization by arc length.

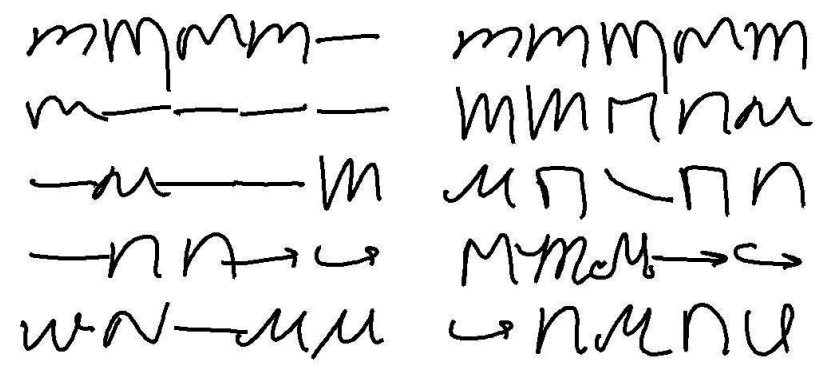

Figure 2. The 25 closest symbols by Legendre (left) and Legendre-Sobolev (right) distance.

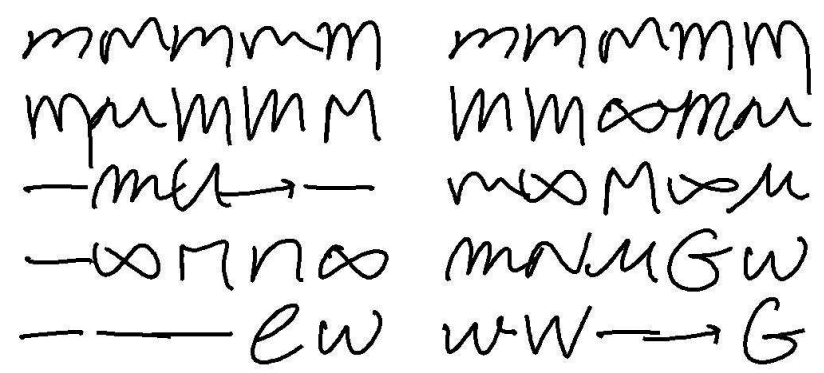

Figure 3. The 25 closest symbols by elastic matching with 10 points (left) and 100 points (right). 
The reason why four decimal digits are sufficient is the following. Assume that $N \times N$ pixels suffice to draw any mathematical character, so that it can be clearly distinguished from all others. In practice, one can safely take $N=1000$. This resolution imposes an intrinsic bound on the precision with which we can compute any numeric feature of a character curve. Namely, the absolute error of any such numeric feature is bounded from below by the amount, by which the feature can vary, if we replace the given curve by another curve that is indistinguishable from given one within our resolution.

The maximal value of a polynomial of degree $d$ on $[-1,1]$, whose Legendre-Sobolev coefficients do not exceed $\epsilon$ in absolute value, is bounded by

$$
\epsilon \sum_{k=1}^{d}\left\|P_{k}(t)\right\|,
$$

where $\left\|P_{k}(t)\right\|$ is the maximum absolute value of the $k$-th normalized Legendre-Sobolev polynomial on [-1, 1$]$. All $\left\|P_{k}(t)\right\|$ can be uniformly bounded by 1 . Therefore, the maximal value of a polynomial of degree $d$, whose coefficients do not exceed $\epsilon$ in absolute value, does not exceed $d \epsilon$.

In order for two curves to be distinguishable on an $N \times N$ screen, their coordinate functions must differ by at least a pixel at some point. If the curves lie within $[-1,1] \times[-1,1]$, and we have 1000 pixels, the size of a pixel is $2 / 1000=0.002$. This implies that $d \epsilon \leq 0.002$ and, for $d=10$, we obtain that $\epsilon=0.0002$ is the maximal precision of the coefficients that we need.

\section{CONCLUSIONS}

Our experiments confirm that the Legendre-Sobolev distance measure is robust enough to serve as a replacement for the elastic matching distance measure, for the purposes of classification of handwritten mathematical characters. The former has a clear advantage in terms of performance, requiring only 60 integer arithmetic operations and being 10-60 times faster than the latter. This means that even an exhaustive linear search for the nearest character with respect to the Legendre-Sobolev distance, over the entire database, can be performed instantly. Moreover, since Legendre-Sobolev distance is a Euclidean distance measure in a 20-dimensional vector space, the search performance can be further improved using kd-trees, Voronoi diagrams, and other efficient indexing techniques that apply to vector spaces.

\section{REFERENCES}

[1] Joshi, N., Sita, G., Ramakrishnan, A. G., and Madhvanath, S., "Comparison of elastic matching algorithms for online tamil handwritten character recognition," in [Proc. Ninth International Workshop on Frontiers in Handwriting Recognition], 444-449 (2004).

[2] Uchida, S. and Sakoe, H., "A survey of elastic matching techniques for handwritten character recognition," IEICE Transactions on Information and Systems E88-D(8), 1781-1790 (2005).

[3] Prasanth, L., Babu, V., Sharma, R., Rao, G. V., and M., D., "Elastic matching of online handwritten tamil and telugu scripts using local features," in [ICDAR '0\%: Proceedings of the Ninth International Conference on Document Analysis and Recognition (ICDAR 2007) Vol 2], 1028-1032, IEEE Computer Society, Washington, DC, USA (2007).

[4] Char, B. W. and Watt, S. M., "Representing and characterizing handwritten mathematical symbols through succinct functional approximation," in [Proc. International Conference on Document Analysis and Recognition (ICDAR)], 1198-1202, IEEE Computer Society (2007).

[5] Golubitsky, O. and Watt, S. M., "Online stroke modeling for handwriting recognition," in [Proc. 18th International Conference on Computer Science and Software Engineering (CASCON 2008)], 72-80 (2008).

[6] Munich, M. E. and Perona, P., "Visual signature verification using affine arc-length," in [1999 IEEE Computer Society Conference on Computer Vision and Pattern Recognition (CVPR'99)], 2 (1999).

[7] Ronveaux, A., "Sobolev inner products and orthogonal polynomials of Sobolev type," Numerical Algorithms 3(1), 393-399 (1992). 